\title{
Expression and Functional Characterization of Retinoic Acid-Inducible Gene-I-Like Receptors of Mast Cells in Response to Viral Infection
}

\author{
Minoru Fukuda ${ }^{a}$ Hiroko Ushio $^{b}$ Junko Kawasaki ${ }^{b, c}$ Francois Niyonsabab \\ Mizuho Takeuchib, c Tadashi Babaa,d Keiichi Hiramatsua,d Ko Okumurab \\ Hideoki Ogawab \\ ${ }^{\mathrm{a}}$ Departments of Infection Control Science, ${ }^{\mathrm{b}}$ Atopy (Allergy) Research Center, and Departments of ${ }^{\mathrm{c}}$ Dermatology \\ and ${ }^{\mathrm{d}}$ Bacteriology, Juntendo University School of Medicine, Tokyo, Japan
}

\section{Key Words}

Antiviral responses - Mast cells $\cdot$ Retinoic acid-inducible gene-l-like receptors $\cdot$ Toll-like receptors $\cdot$ Virus recognition

\begin{abstract}
To investigate the precise mechanisms of virus recognition by mast cells, the expression and functional characteristics of virus recognition receptors that lead to mast cell activation were investigated. Our results suggest that mast cells are partly responsible for the early in vivo production of antiviral cytokines and chemokines upon vesicular stomatitis virus (VSV) infection. Analysis of the expression of doublestranded RNA (dsRNA) recognition receptors in murine bone marrow-derived mast cells (BMMCs) revealed that BMMCs express melanoma differentiation-associated gene 5 (MDA5), protein kinase RNA-activated, retinoic acid-inducible gene-I (RIG-I) and Toll-like receptor 3. The expression levels of these receptors were found to increase upon stimulation of mast cells with VSV as well as synthetic dsRNA: polyinosinic-polycytidylic acid. Moreover, small interfering RNA analysis to identify the receptors responsible for mast cell activation by VSV revealed that both RIG-I and MDA5 were involved in cytokine production but not in the degranulation of mast cells. Our findings suggest that mast cells pro-
\end{abstract}

duce cytokines and chemokines in the early infection stage after recognizing viruses via RIG-I and MDA5, and may contribute to antiviral responses. These data provide additional novel information that improves our understanding of antiviral innate responses that involve mast cells.

Copyright $\odot 2012$ S. Karger AG, Basel

\section{Introduction}

Mast cells are recognized as key effector cells in IgEassociated allergic diseases, and recent accumulating evidence suggests that these cells are also among the central players in the innate immune response [1]. The recognition of pathogens by mast cells takes place through two major pathways: via the direct binding of pathogens by pattern recognition receptors (PRRs) or via the binding of antibody- and complement-coated bacteria to the $\mathrm{Fc}$ and complement receptors on the mast cell surface. PRRs are necessary for the protection of the host against microbial infections and trigger the innate immune response after sensing microbial components, such as lipopolysaccharide (LPS), lipoprotein, flagellin and nucleic acids. The coordinated activation of transcription factors triggered by the recognition of microbial components by PRRs results

\section{KARGER \\ Fax +4161306 1234 \\ E-Mail karger@karger.ch}

www.karger.com
(C) 2012 S. Karger AG, Basel

$1662-811 X / 13 / 0052-0163 \$ 38.00 / 0$

Accessible online at:

www.karger.com/jin
Dr. Hiroko Ushio

Atopy Research Center, Juntendo University School of Medicine

2-1-1 Hongo, Bunkyo-ku

Tokyo 113-8421 (Japan)

E-Mail hushio@juntendo.ac.jp 
in the expression of inflammatory cytokines, chemokines and type I interferons (IFNs). Although many studies suggest that mast cells contribute to innate immune responses against invading bacteria, the knowledge regarding the role of mast cells in viral responses is limited.

Several in vitro studies have suggested that virus or virus products stimulate mast cells to produce cytokines and chemokines, or to release its granular contents [2-5]. In support of direct viral recognition by mast cells, it has been shown that histamine release and cytokine production can occur in response to Sendai virus and the HIV gp120 envelope protein, respectively [3, 4]. Furthermore, the specific production of antiviral cytokines, such as type I IFN, has been observed in mast cells stimulated with a virus or polyinosinic-polycytidylic acid (poly I:C) $[6,7]$. Orinska et al. [8] reported the functional consequences of mast cell activation in response to viral infection; they demonstrated that mast cells, when stimulated via Toll-like receptor (TLR) 3, produced chemokines that mediated CD8+ T-cell recruitment in vivo. Furthermore, it has been reported that the TLR-induced activation of mast cells by LPS or poly I:C enhanced the capacity of mast cells to activate CD8+ T cells [9]. Thus, the recognition of viruses by mast cells may promote cell-mediated responses that lead to intracellular virus clearance.

Innate immune responses against viral infection begin with the recognition of the virus by a specific PRR. Viral double-stranded RNA (dsRNA) is one of the most important viral structures involved in the activation of immune responses. Recent advances in the identification of pathogen receptors in the innate immune system have revealed that distinct types of sensors play a role in the detection of viral nucleic acids by various mechanisms: TLRs detect viral DNA or RNA in the endosomal compartment of immune cells; retinoic acid-inducible gene-I (RIG-I)-like receptors (RLRs) recognize viral RNA in the cytoplasm, and DNA sensors detect cytoplasmic viral DNA [10]. Human and rodent mast cells have been shown to express PRRs, such as TLR1-TLR7 and TLR9, that respond to specific ligands by inducing the production of cytokines or chemokines or the release of the granular cell contents [11, 12]. Some, but not all mast cells, have been reported to express TLR3 and TLR7 and to respond to poly I:C and TLR7 ligands, which are thought to be produced by viruses $[6,13]$. Poly I:C is a synthetic analog of dsRNA and is widely used to mimic viral infection. However, the recognition of poly I:C by TLRs and the downstream cellular responses are highly variable between cells, and the responses to poly I:C are not always representative of the responses of actual viral infections. Furthermore, the in- tracellular expression of RLRs and their relative contribution to the responsiveness of mast cells to viruses remain to be elucidated. In this study, we examined RLR expression in mast cells and the contribution of these receptors to the antiviral responses of mast cells.

\section{Materials and Methods}

Mice

TLR3-/- mice, which were originally described by Alexopoulou et al. [14], were kindly provided by Dr. Flavell and kept in our animal facility. Genotyping of animals was performed by PCR of DNA obtained from tail biopsies. Primers were created according to the genotyping protocol (stock No. 005217) from the Jackson Laboratory (Bar Harbor, Me., USA). The following primers were used: forward primer 5'-GCC AGA GGC CAC TTG TGT AG-3' for the detection of both wild and mutated type, and reverse primers 5'-GCA ACC CTT TCA AAA ACC AG-3' for the detection of wild type and 5'-AAT TCA TCA GTG CCA TGA GTT T-3' for the detection of mutated type. Mast cell-deficient $\mathrm{Kit}^{\mathrm{W}-\mathrm{sh} / \mathrm{W}-\mathrm{sh}}$ mice with C57BL/6 background were provided by S. Nakae (Institute of Medical Science, University of Tokyo, Japan), and wildtype C57BL/6 mice were purchased from Japan Clea (Tokyo, Japan). Bone marrow cells were prepared from 8- to 12-week-old mice as described below and used for bone marrow-derived mast cell (BMMC) induction. All experiments were performed according to the guidelines approved by the Institutional Animal Experiments Committee of the Juntendo University School of Medicine.

\section{Viral Infection}

Vesicular stomatitis virus (VSV) was kindly provided by Drs. Honda and Yanai (University of Tokyo), and $1 \times 10^{7}$ PFU of virus was used to intravenously infect 7 - to 8 -week-old mice. Serum was collected $6 \mathrm{~h}$ after infection according to the preliminary experiment. In some experiments, the mice were followed up for 2 weeks to determine survival.

\section{Cell Culture and Murine BMMC Stimulation}

BMMCs were generated as described previously [12]. Briefly, bone marrow cells were cultured for 5- 8 weeks in RPMI 1640 medium (Sigma-Aldrich, Tokyo, Japan) supplemented with $10 \%$ heatinactivated FCS, $100 \mu \mathrm{M}$ 2-ME, $10 \mu \mathrm{M}$ MEM nonessential amino acids, $100 \mathrm{U} / \mathrm{ml}$ penicillin, $100 \mu \mathrm{g} / \mathrm{ml}$ streptomycin and $10 \mathrm{ng} / \mathrm{ml}$ murine IL-3 (Peprotech, Rocky Hill, N.J., USA). After 5 weeks of culture, cells that were $>98 \%$ positive for both c-kit and FceRI on the cell surface, as determined by flow cytometry, were classified as mature mast cells. Cells $\left(1 \times 10^{6}\right.$ cells/ml $)$ were stimulated with VSV at the indicated multiplicities of infections (MOIs) for $8 \mathrm{~h}$ for mRNA expression and $24 \mathrm{~h}$ for cytokine production. All these time points were determined by preliminary experiments. In some experiments, the virus was irradiated with UV light for 30 min while on ice and then used for experiments. Poly I:C of two differently sized strands (all from InvivoGen, San Diego, Calif., USA) were used for stimulation of BMMCs at the concentrations indicated in the figure legends. Poly I:C(H) with a high molecular weight has an average size of $1.5-8 \mathrm{~kb}$. Poly I:C(L) with a low molecular weight 
has an average size of $0.2-1 \mathrm{~kb}$. Polyadenylic-polyuridylic acid (poly A:U; InvivoGen), a putative TLR3 ligand that signals only through TLR3, was also used. For IgE cross-linking stimulation, BMMCs were sensitized for $1 \mathrm{~h}$ with $1 \mu \mathrm{g} / \mathrm{ml}$ anti-TNP IgE monoclonal antibody (clone IgE-3; BD Biosciences, San Jose, Calif., USA) and then stimulated with anti-IgE antibody $(0.5 \mu \mathrm{g} / \mathrm{ml}$; BD Bioscience) for $6 \mathrm{~h}$ [12]. In some experiments, BMMCs were also stimulated with LPS ( $1 \mu \mathrm{g} / \mathrm{ml}$; Sigma, Tokyo, Japan) for $6 \mathrm{~h} \mathrm{[12].} \mathrm{To}$ analyze the degranulation of mast cells, mast cells were resuspended at $1 \times 10^{6}$ cells $/ \mathrm{ml}$ in Tyrode's buffer containing $0.1 \%$ BSA and stimulated with VSV at the MOIs indicated, or IgE-sensitized mast cells were stimulated with anti-mouse IgE (BD Bioscience) for 1 or $6 \mathrm{~h}$. The release of $\beta$-hexosaminidase was measured as previously described [12]. Degranulation was expressed as the percent release of $\beta$-hexosaminidase: (OD of the stimulated supernatant - OD of the unstimulated supernatant/OD of the total lysate - OD of the unstimulated supernatant) $\times 100 \%$, with $\mathrm{OD}=$ optical density.

\section{Quantitative PCR}

Total RNA was extracted from cells using the RNAspin Mini kit (GE Healthcare, Chalfont St Giles, UK) and treated with DNase I (GE Healthcare) and the RNeasy Plus Mini kit (Qiagen, Hilden, Germany). Complementary DNA was synthesized with SuperScript ${ }^{\mathrm{TM}}$ II reverse transcriptase (Invitrogen, Tokyo, Japan) using random primers. Quantitative PCR was performed with a TaqMan method using the ABI 7500 system (Applied Biosystems, Piscataway, N.J., USA). mRNA levels were normalized to glyceraldehyde 3-phosphate dehydrogenase gene expression and expressed as fold induction relative to uninfected cells.

\section{Measurement of Cytokines}

BMMCs $\left(1 \times 10^{6} / \mathrm{ml}\right)$ were stimulated with the indicated concentrations of virus for $24 \mathrm{~h}$. IFN- $\alpha$ and IFN- $\beta$ concentrations in the supernatants were determined using ELISA kits according to the manufacturer's instructions (PBL Interferon Source, Piscataway, N.J., USA). The interleukin (IL)-6, inducible protein (IP)-10, macrophage inflammatory protein (MIP) $1 \beta$ and monocyte chemoattractant protein (MCP) 1 concentrations in the supernatants or serum were also determined using ELISA kits according to the manufacturer's instructions (R\&D Systems, Minneapolis, Minn., USA).

\section{Western Blotting}

BMMCs were untreated or infected with VSV (MOIs = 3) for $24 \mathrm{~h}$. After stopping the reaction by adding ice-cold PBS, sample buffer containing SDS was directly added to the pellets followed by brief sonication. Then, lysates were subjected to SDS-PAGE and transferred onto polyvinylidene difluoride membranes (Millipore, Billerica, Mass., USA). The membranes were analyzed by immunoblotting with anti-melanoma differentiation-associated gene 5 (MDA5), anti-RIG-I (both from Cell Signaling Technology, Danvers, Mass., USA) or anti- $\beta$-actin (BioLegend, San Diego, Calif., USA), followed by the respective HRP-conjugated, secondary, anti-immunoglobulin antibodies (GE Healthcare). The membranes were developed with Luminata ${ }^{\mathrm{TM}}$ Forte Western HRP substrate (Millipore).

Knockdown of RLRs by Small Interfering RNA

BMMCs were transfected with control or specific small interfering RNA (siRNA) for $p k r$, rig- $I, m d a 5$ and $t$ tr3 (Applied Biosys-

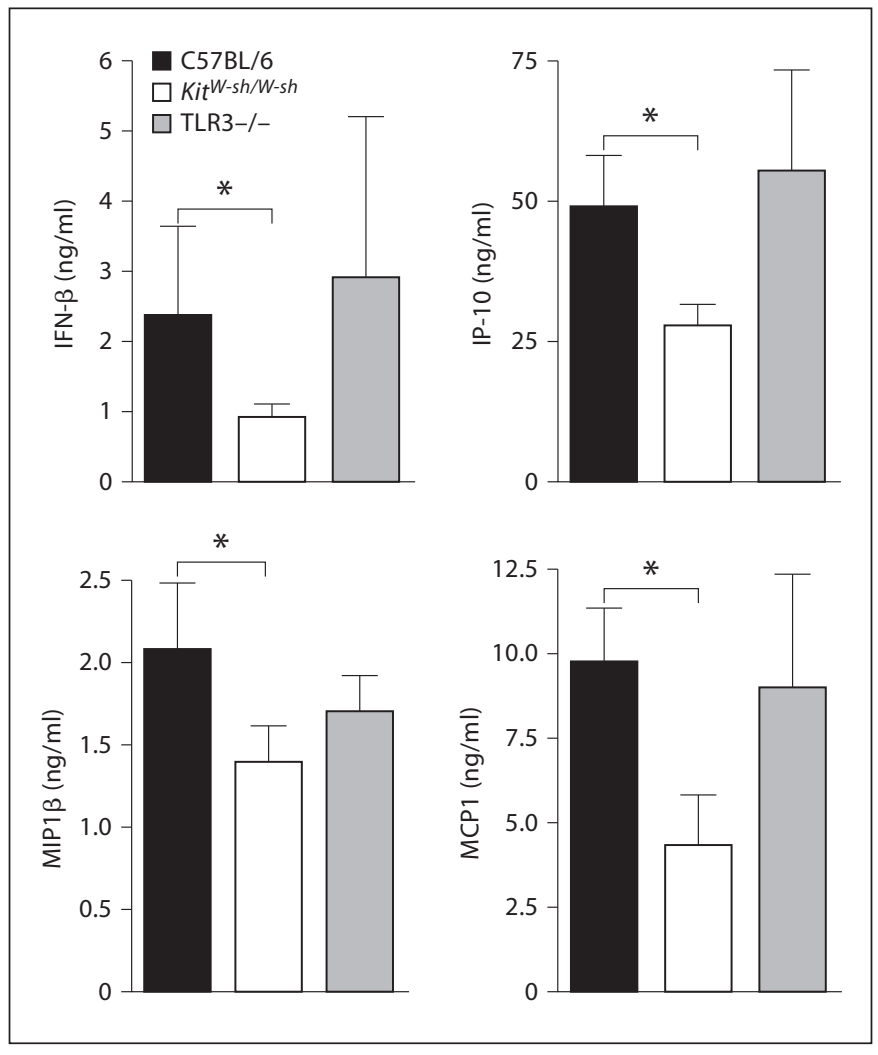

Fig. 1. Mast cells are partly responsible for the cytokine production upon VSV infection in vivo. VSV $\left(1 \times 10^{7} \mathrm{PFU}\right)$ was intravenously administered to 7 - to 8 -week-old mice and serum was collected $6 \mathrm{~h}$ after infection; cytokine concentrations in the serum were measured by ELISA. Means \pm SD of $4-5$ mice. ${ }^{*} p<0.05$ vs. wild-type C57BL/6 mice.

tems) using the Nucleofector system (Amaxa; program T-30, solution V, Lonza, Basel, Switzerland). The individual gene silencing efficiency was determined by quantitative PCR $24 \mathrm{~h}$ after transfection, and the cells were then used for experiments.

\section{Statistical Analysis}

Non-parametric Mann-Whitney's U test was used. Values of $\mathrm{p}<0.05$ were regarded as statistically significant.

\section{Results}

\section{Mast Cells Are Partly Responsible for the Cytokine}

Production upon VSV Infection in vivo

To examine the role of mast cells and TLR 3 in antiviral responses, we infected mast cell-deficient $\mathrm{Kit}^{\mathrm{W} \text {-sh/W-sh }}$ mice and TLR3-deficient mice with VSV and measured antiviral cytokine and chemokine levels produced $6 \mathrm{~h}$ after VSV infection (fig. 1). Preliminary experiments 

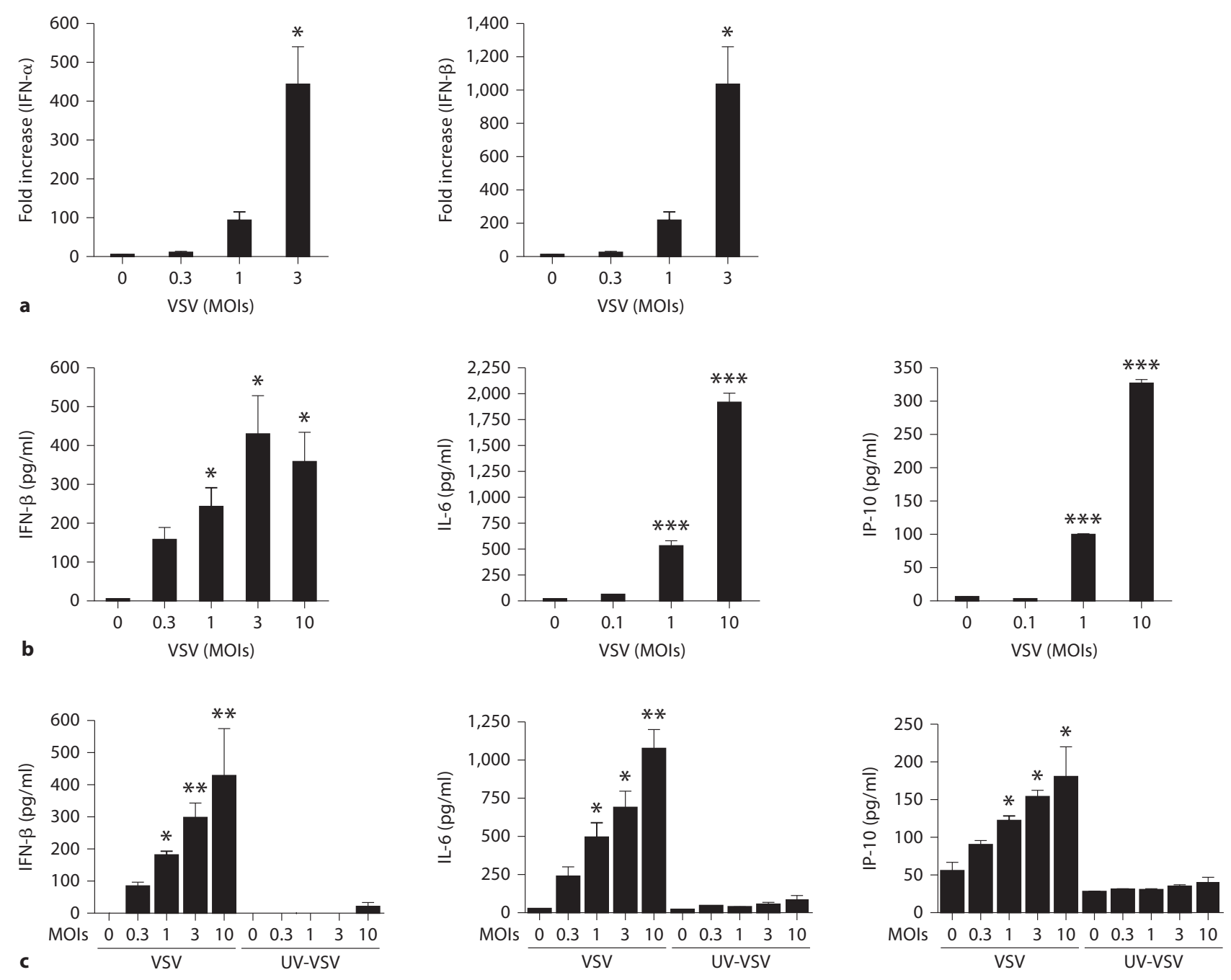

Fig. 2. Viral infection directly causes antiviral responses in mast cells in vitro. a BMMCs were stimulated with various concentrations of VSV for $8 \mathrm{~h}$. The levels of mRNA for IFN- $\alpha$ and IFN- $\beta$ were determined by quantitative-PCR. $\mathbf{b}$ The levels of secreted IFN- $\beta$, IL- 6 and IP-10 in the supernatant of cultures stimulated with the indicated concentrations of VSV for $24 \mathrm{~h}$ were measured by ELISA. $\mathbf{c}$ IFN- $\beta$, IL- 6 and IP-10 production upon stimulation with the indicated concentrations of VSV or UV-irradiated VSV for $24 \mathrm{~h}$ were measured by ELISA. Means \pm SD of 3-5 separate experiments. ${ }^{*} \mathrm{p}<0.05,{ }^{* *} \mathrm{p}<0.01,{ }^{* * *} \mathrm{p}<0.001$, vs. unstimulated cells. showed that cytokine production peaked $6 \mathrm{~h}$ after VSV infection and gradually decreased during the next $24 \mathrm{~h}$ (data not shown). The levels of antiviral cytokines, IFN- $\beta$ and several chemokines were significantly lower but not completely abolished in the mast cell-deficient $\mathrm{Kit}^{\mathrm{W}-\mathrm{sh} / \mathrm{W} \text {-sh }}$ mice. In contrast, cytokine production in the TLR3-deficient mice was comparable to production in the wild-type mice. These results suggest that mast cells are responsible for the early cytokine production in vivo upon VSV infection, independent of TLR3 involvement.
Although mast cell-deficient mice produced lower amounts of antiviral cytokines, the survival rates of these mice, including the TLR3-deficient mice, did not differ 2 weeks after VSV infection (data not shown).

\section{Viral Infection Directly Causes Antiviral Responses in Mast Cells in vitro}

To investigate whether viral infection directly leads to antiviral cytokine production in mast cells, we first cultured mast cells with VSV. VSV infection caused both 
IFN- $\alpha$ and IFN- $\beta$ induction in mast cells in a dose-dependent manner (fig. 2a). Cytokine production was also detectable at the protein level when measured by ELISA (fig. 2b). In addition to type I IFN, BMMCs also produced IL-6, IP-10, MCP1 and MIP1 $\beta$, corroborating the in vivo data (fig. $2 \mathrm{~b}$ and data not shown). Intriguingly, mast cells did not produce any cytokines when exposed to UV-irradiated VSV, suggesting that only live virus can induce mast cells to produce cytokines (fig. 2c).

\section{TLR3 Is Not Required for Antiviral Cytokine \\ Responses in Mast Cells}

Given that mast cells were activated by live VSV, we next determined which receptors are responsible for the recognition of viruses. Several receptors are implicated in the recognition of viruses and the subsequent activation of various cells [10]. We generated BMMCs from TLR3deficient mice, which were stimulated with increased VSV concentrations. In agreement with the in vivo results, TLR3-deficient BMMCs produced IFN- $\beta$ levels comparable to those produced by wild-type BMMCs following infection with VSV, suggesting that TLR3 is not required for the induction of antiviral cytokine responses in mast cells (fig. 3). We lack evidence demonstrating that TLR3 plays a critical role in the activation of mast cells because we were unable to stimulate BMMCs with poly I:C, as measured by cytokine levels. Additionally, because we did not detect significant differences in cytokine production in vivo between the wild-type and TLR3deficient mice upon VSV infection (fig. 1), TLR3 may not be involved in VSV recognition by mast cells or other cell types.

\section{Mast Cells Express Several RLRs and the Expression}

Levels of These RLRs Are Upregulated by Viral

Infection

Several intracellular receptors against dsRNA have been implicated in other cell types. Thus, we examined whether these receptors also play a role in the recognition and activation of mast cells by VSV. Resting mast cells express mRNA for MDA5, protein kinase RNA-activated (PKR) and RIG-I (fig. 4a). TLR3 mRNA was almost undetectable. mRNA levels of each receptor increased drastically upon VSV infection. The greatest response was observed for RIG-I (fig. 4b). Interestingly, TLR3 mRNA was slightly but statistically significantly increased by VSV infection (fig. 4b). Receptor expression was also confirmed at protein level by Western blotting (fig. 4c). Consistent with mRNA results, increased expression of MDA5 and RIG-I were obvious at protein levels upon vi-

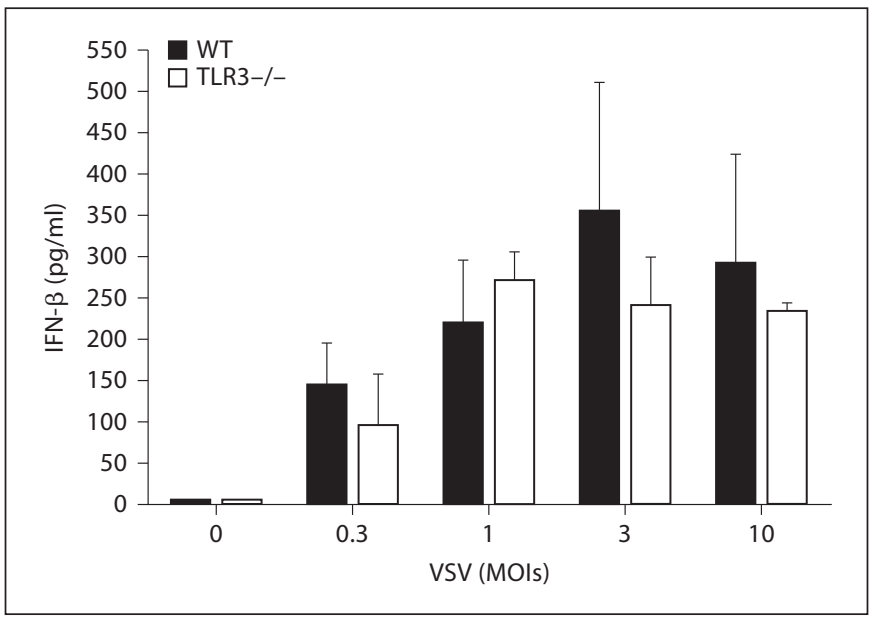

Fig. 3. TLR3 is not required for antiviral cytokine responses in mast cells. BMMCs from wild-type (WT) or TLR3-deficient mice were stimulated with the indicated concentrations of VSV. The level of IFN- $\beta$ in the supernatant $24 \mathrm{~h}$ after stimulation was investigated by ELISA. Means \pm SD of 3 separate experiments.

rus infection. Although poly I:C did not induce mast cells to produce cytokines, poly I:C [both $(\mathrm{H})$ and $(\mathrm{L})$ forms], but not the putative TLR3 ligand poly A:U, produced a modest but statistically significant increase in mRNA levels for several dsRNA receptors, including TLR3 (fig. $4 \mathrm{~d}$ and data not shown). Based on previous evidence indicating that long and short forms of dsRNA are recognized by MDA5 and RIG-I, respectively [15], and since poly A:U had no effect on the expression of dsRNA receptors of BMMC, mast cells may be partially responsive to poly I:C via receptors other than TLR3.

Both MDA5 and RIG-I Are Required for the Induction of Antiviral Cytokine Production by Mast Cells in

Response to VSV Infection

To identify the receptors responsible for the recognition and activation of mast cells after VSV infection, we treated BMMCs with siRNA for the respective receptors and examined the antiviral cytokine responses. First, the specific knockdown of each receptor by the corresponding siRNA was confirmed (fig. 5a). Then, BMMCs transfected with specific siRNA were stimulated with VSV, and cytokine production was assessed. We found that siRNA inhibition of MDA5 and RIG-I, but not PKR or TLR3, significantly reduced the level of cytokine production by mast cells in response to VSV (fig. 5b). The suppressive effect of siRNA treatment was restricted to antiviral responses because cytokine responses to a TLR4 li- 


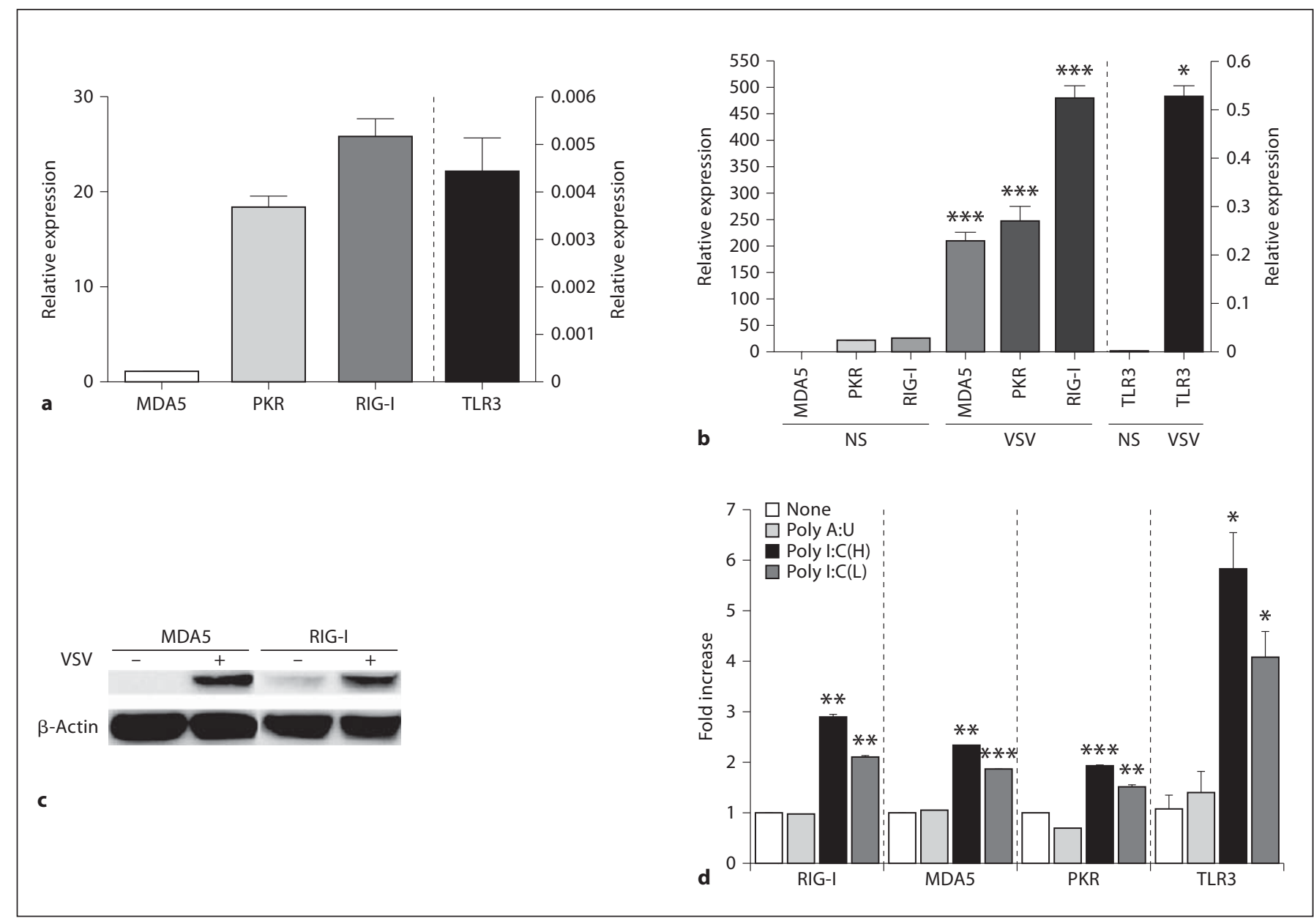

Fig. 4. Mast cells express several RLRs, and expression levels of these RLRs are upregulated by viral infection. mRNA levels of MDA5, RIG-I, PKR and TLR3 in unstimulated BMMCs (a) or BMMCs stimulated with VSV (MOIs $=3$; b) for $8 \mathrm{~h}$ were quantified by real-time PCR and expressed as relative expression levels based on the MDA-5 level (MDA5 = 1). NS = No stimulation. c MDA5 and RIG-I expression at protein levels in unstimulated or VSV (MOIs $=3$ )-stimulated-BMMC for $24 \mathrm{~h}$ were analyzed by

gand, LPS and IgE cross-linking were not altered in the mast cells treated with siRNAs against these receptors (fig. 5c).

\section{VSV Infection Induces Degranulation of Mast Cells}

Independent of Known Sensors for the dsRNA

Because mast cell activation is often associated with degranulation, we examined whether VSV infection leads to mast cell degranulation. Although we could not detect significant $\beta$-hexosaminidase release $1 \mathrm{~h}$ after VSV infection, a modest but statistically significant re-
Western blotting. Equal loading of protein in each lane was confirmed by reprobing the same membrane with anti- $\beta$-actin. $\mathbf{d}$ mRNA levels of MDA5, RIG-I, PKR and TLR3 in BMMCs $8 \mathrm{~h}$ after stimulation with $10 \mu \mathrm{g} / \mathrm{ml}$ of poly A:U, poly I:C(H) and poly I:C(L) were quantified by real-time PCR and expressed as relative expression levels based on the expression levels of each of the receptors in unstimulated mast cells. Means \pm SD of 3 experiments. ${ }^{*} \mathrm{p}<0.05,{ }^{* *} \mathrm{p}<0.01,{ }^{* *} \mathrm{p}<0.001$, vs. unstimulated cells. lease of $\beta$-hexosaminidase was observed $6 \mathrm{~h}$ after VSV infection (fig. 6a). In contrast to the results for cytokine production, the degranulation caused by VSV $6 \mathrm{~h}$ after infection was not inhibited by siRNA irrespective of the receptor type (fig. 6b), suggesting that the degranulation of mast cells by VSV might be mediated by different RNA sensors or by viral products whose recognition is independent of these receptors. 
Fig. 5. Both MDA5 and RIG-I are required for the induction of antiviral cytokine production by mast cells in response to VSV infection. a siRNA was used to knock down endogenous PKR, MDA5, RIG-I and TLR3 as described in the Methods. The effects of siRNAs were confirmed by real-time PCR $24 \mathrm{~h}$ after transfection. b BMMCs were stimulated with VSV $24 \mathrm{~h}$ after the corresponding siRNA transfection. IFN- $\alpha$, IFN- $\beta$, IL- 6 and IL-10 levels were determined by ELISA $24 \mathrm{~h}$ after VSV infection. c IL-6 production upon stimulation with LPS or IgE cross-linking was measured by ELISA $6 \mathrm{~h}$ after stimulation. Means \pm SD of 3 separate experiments. ${ }^{*} \mathrm{p}<0.05,{ }^{* *} \mathrm{p}<0.01,{ }^{* * *} \mathrm{p}<0.001$, vs. control siRNA-treated cells.

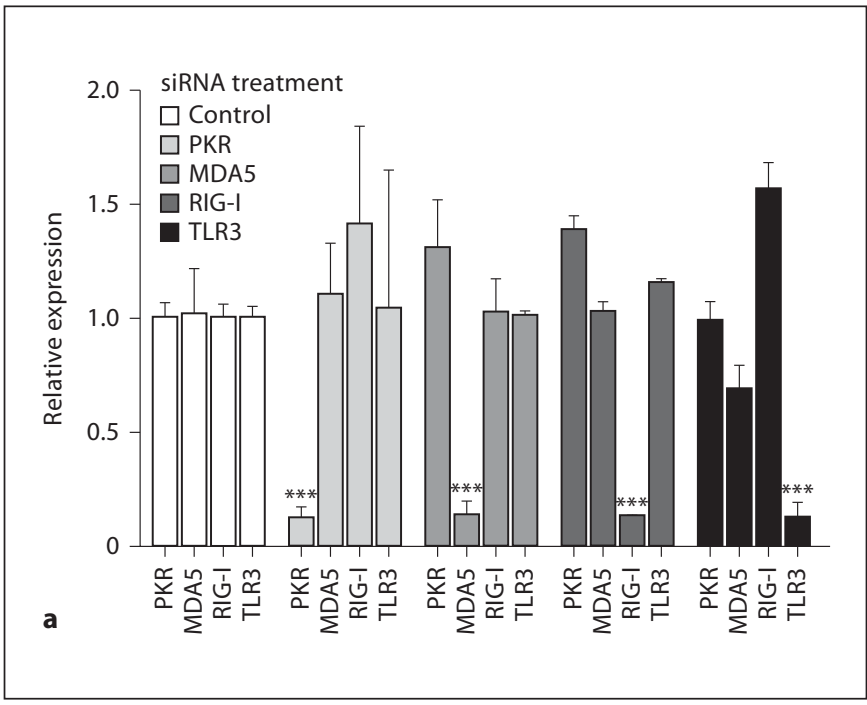

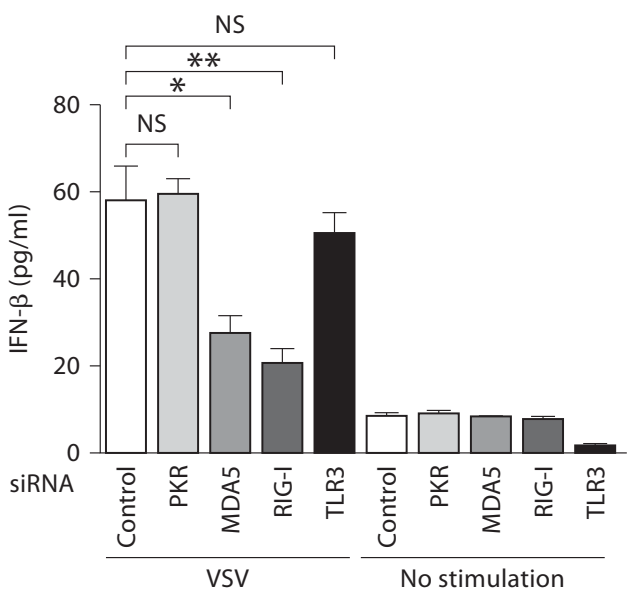

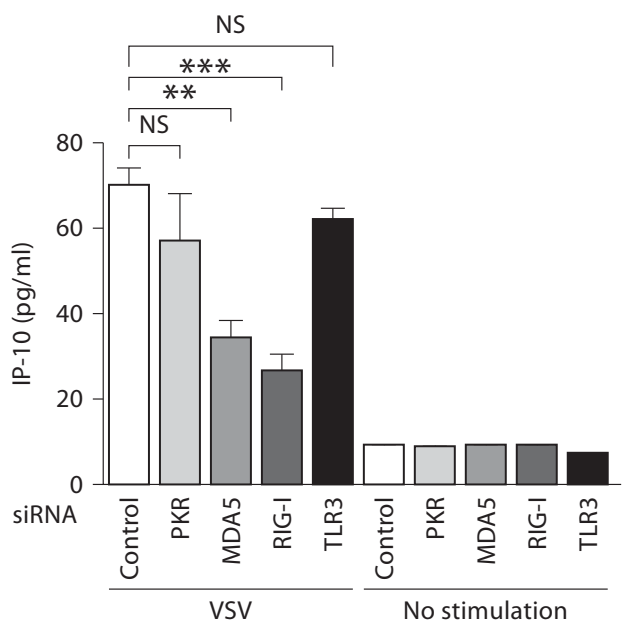

For figure $5 c$ see next page. 

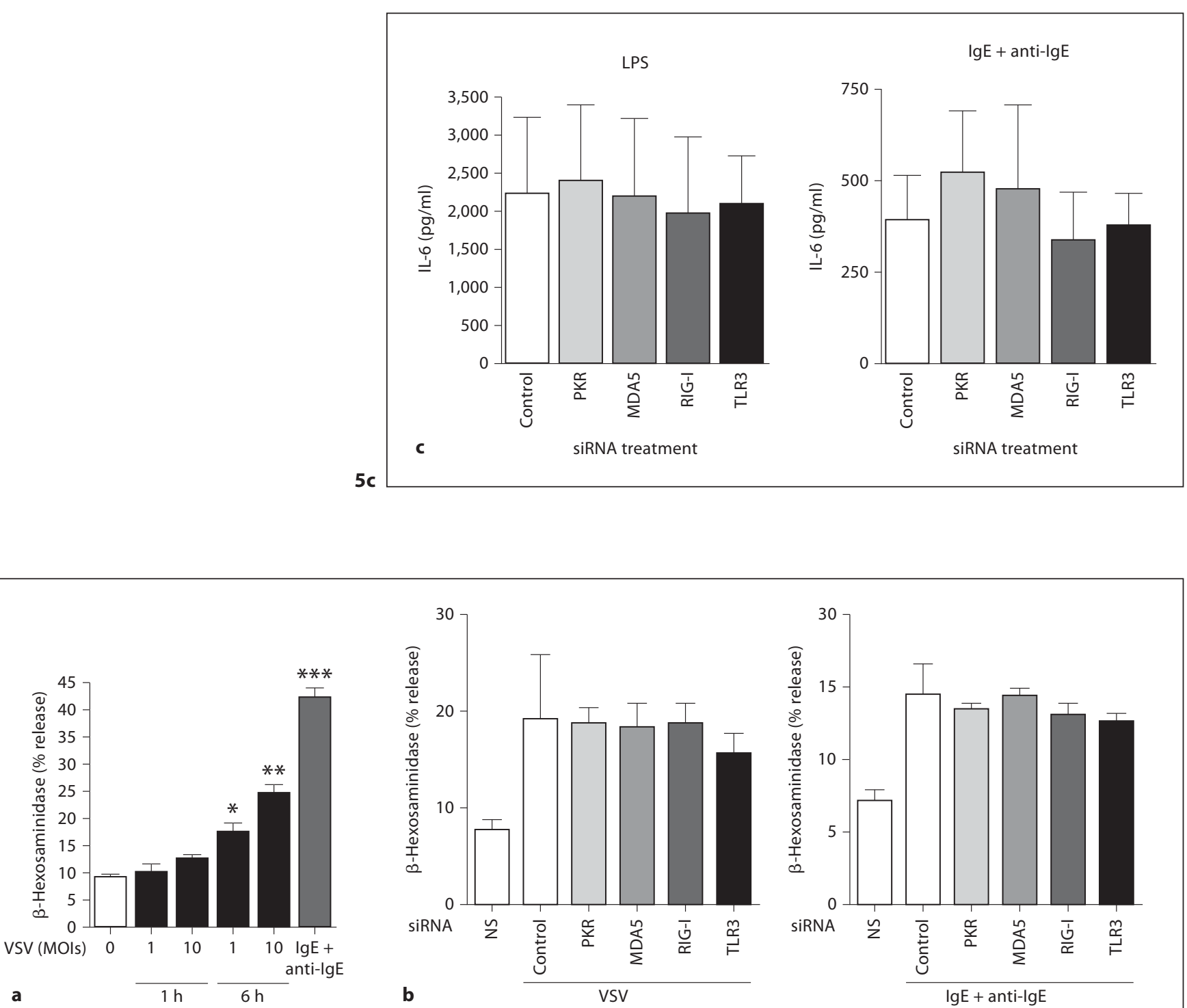

Fig. 6. VSV infection induces the degranulation of mast cells independent of known sensors for the dsRNA. a The release of $\beta$ hexosaminidase from BMMCs stimulated with the indicated concentrations of VSV for 1 or $6 \mathrm{~h}$ was measured as described in the Methods. The release of $\beta$-hexosaminidase by IgE-sensitized and anti-IgE-stimulated mast cells served as a positive control. b siRNA was used to knock down endogenous PKR, MDA5, RIGI and TLR3 as described in the Methods. BMMCs were stimulated with VSV for $6 \mathrm{~h}$, and the release of $\beta$-hexosaminidase was assessed as described in the Methods. ${ }^{*} \mathrm{p}<0.05,{ }^{* *} \mathrm{p}<0.01$, ${ }^{* * *} \mathrm{p}<0.001$, vs. unstimulated cells. NS $=$ No stimulation.

\section{Discussion}

Given the fact that mast cells are located in almost all tissues and are especially numerous in organs that interface with the environment, it is not surprising that they participate in immune surveillance. The role of mast cells in immune surveillance and innate immunity against bacterial pathogens has been well defined [16]. However, studies on the mechanisms inducing mast cell responses to live viruses are limited.

In this experiment, we demonstrated a modest but statistically significant reduction in the antiviral cytokine and chemokine responses in mast cell-deficient $\mathrm{Kit}^{\mathrm{W}-\mathrm{sh} / \mathrm{W} \text {-sh }}$ mice but not in TLR3-deficient mice upon 
VSV infection. Because VSV infection was not lethal even at high doses, we could not analyze the differences in the survival rate between the mast cell-deficient and mast cell-competent mice. However, a recent study using a mouse model of infection with dengue virus and Newcastle disease virus demonstrated that mast cells play an important role in the recruitment of natural killer (NK) cells, NK1.1+ T cells and CD8+ T cells to the site of viral infection by inducing early antiviral cytokine and chemokine production $[8,17]$. Thus, both the nature of mast cells as tissue-resident cells and their ability to directly recognize and respond quickly to a virus by producing antiviral cytokines and chemokines make mast cells key players of the immune responses during the early stages of viral infection. The lack of TLR3 involvement in antiviral cytokine responses was consistent with previous results obtained with VSV-infected TLR3-deficient mice; experiments with these mice demonstrated the generation of an effective antiviral response was independent of TLR3 [18]. Because mast cell activation is required for the recruitment of $\mathrm{CD} 8+\mathrm{T}$ cells to the site of viral infection and because TLR3-mediated signaling is implicated in the cross-priming of cytotoxic CD8+ T cells by plasmacytoid dendritic cells during viral infection $[8,19]$, TLR3mediated systems provide different contributions depending on the type of virus and the route of infection.

Although it has been repeatedly assumed that TLR3 plays an important role in the activation of human and rodent mast cells by poly I:C and several viruses $[6,13]$, the roles played by the cytoplasmic receptors for dsRNA during viral recognition by mast cells have not been fully investigated. We detected high expression of RIG-I and almost undetectable levels of TLR3 in resting mast cells (fig. 4). The low level of TLR3 expression and the limited response to poly I:C might be due to the immaturity of our mast cells because fetal skin-derived murine mast cells have been reported to express TLR 3 and be able to respond to poly I:C [13]. It has also been reported that the ability of human mast cells to respond to poly I:C, in either the extracellular or cytosolic form, varied among the populations of mast cells examined [20].

RLRs are broadly expressed in most tissues, and their expression is typically maintained at low levels in resting cells but is greatly increased after IFN exposure and viral infection $[21,22]$. We observed a marked increase in the expression of these receptors, including PKR and TLR3, after VSV infection. Additionally, a recent report documented upregulation of mRNA and protein levels of the RNA sensors RIG-I and MDA5 and of multiple IFNstimulated genes in human mast cells in response to an- tibody-enhanced dengue virus infection [20]. Interestingly, poly I:C, which failed to stimulate cy tokine production by BMMCs in our system, induced the upregulation of three intracellular receptors and TLR3, albeit to a much smaller degree compared to the actual viral infection, suggesting that the mast cells in our study are somehow able to respond to poly I:C. Since both MDA5 and RIG-I are reported to participate in the length-dependent recognition of poly I:C [15], and the putative TLR3-ligand poly A: $\mathrm{U}$ did not induce an increase dsRNA receptors of BMMC, these BMMC responses to poly I:C might be mediated by other receptors than TLR3.

It has been reported that viral genomes and replication products are the main triggers of PRRs, such as TLR3, TLR7 and TLR9, which sense dsRNA, single-stranded RNA (ssRNA) and DNA, respectively, delivered into the endosomes during viral infection. Specialized plasmacytoid dendritic cells produce type I IFNs when TLR7, TLR8 and TLR9 are triggered by RNA or DNA viral genomes in the endosomes [23]. In contrast, most cells rely on RLRs, the cytosolic sensors that detect RNA that accumulates in the cytoplasm during viral infection [10, 22]. VSV, a member of the Rhabdovirus family, contains a negative-sense ssRNA genome. The mechanism by which the innate immune system detects VSV has been thoroughly investigated [24-28]: RIG-I mediates a specific response to VSV as well as many other negativestrand viruses, such as influenza and Sendai viruses, and some positive-strand RNA viruses, such as Japanese encephalitis virus [29]. Although 5' triphosphorylated RNA, which is present in the genomes of influenza and other negative-strand RNA viruses but not picornaviruses, has been shown to preferentially stimulate RIG-I [28, 30,31 , the existence of other products capable of specifically stimulating RIG-I has not been verified. We demonstrated that, in mast cells, antiviral cytokine and chemokine responses against VSV were dependent on both MDA5 and RIG-I but not on TLR3 or PKR. Our results were consistent with the reported human mast cell responses to dengue virus, an ssRNA virus from the genus Flavivirus; the knockdown of RIG-I and MDA5 but not TLR3 resulted in the loss of cellular antiviral responses to dengue [17]. Although some viruses such as dengue virus and West Nile virus are reported to be sensed by both RIG-I and MDA5 [32], the exact mechanism by which these viruses activate MDA5 remains unknown. Moreover, although it is generally assumed that MDA5 can be activated by long dsRNAs which originate from positive-strand RNA viruses such as picornavirus, DNA viruses and synthetic RNA poly I:C $[15,28]$, the existence 
of specific ligands for MDA5 in picornaviruses has not been identified. Interestingly, a recent report suggested that MDA5 can be activated by higher-order structured RNA that contains ssRNA and dsRNA, rather than simply long molecules of dsRNA [31]. Thus, the dependency of different RLRs to recognize different viruses seems variable among cells and may provide different contributions depending on the route of infection.

The innate immune response to a viral infection is characterized by the rapid production of a number of cytokines, with type I IFN being the most abundant. Previously, IFN- $\alpha$ production by human mast cells was suggested to be specific to viral stimuli in the context of TLR activation [7]. Chemokines such as CCL3, CCL4 and CCL5 are also important for the trafficking of leukocytes, such as $\mathrm{T}$ and NK cells, which are thought to play important roles in viral infections. The differential release of mediators in response to various triggers is a pivotal event underlying the biology and function of mast cells in host defense and in the pathogenesis of various diseases. Most pathogens appear to induce lipid mediator and cytokine secretion and not degranulation [11]. However, VSV induced the degranulation of mast cells after $6 \mathrm{~h}$ but not after $1 \mathrm{~h}$ of infection, suggesting that viral infection is necessary to induce mast cell degranulation. This result is consistent with the cytokine responses to live viruses, the replication of which requires several hours after infection. In contrast, a study using dengue virus demonstrated that viral particles did not need to be infectious to cause mast cell degranulation because there was no observable difference in the degree of degranulation be- tween mast cells exposed to live and UV-inactivated virus [17]. Remarkably, we did not observe inhibition of degranulation by VSV following transfection with siRNAs against each receptor, suggesting that VSV causes mast cell degranulation through the activation of receptors other than MDA5, RIG-I, PKR and TLR3. Further studies are required to determine the mechanisms of virusrecognition that lead to mast cell degranulation.

Together with the fact that mast cells are located at the body surfaces that interface the external environment, our findings expand the defined role of immunosurveillance by mast cells to include viral pathogens. Additionally, taking into account the fact that both allergens and microbial antigens can trigger mast cell mediator release and that these triggering pathways are integrated, there is a possibility that the mast cell virus interaction is potentially detrimental for the host. Thus, increased understanding of the molecular mechanisms underlying these processes could offer new strategies for immune and antiviral therapy by targeting the RLR pathway in mast cells.

\section{Acknowledgments}

This work was supported in part by a MEXT-Supported Program for the Strategic Research Foundation at Private Universities [Atopy (Allergy) Research Center, Juntendo University]. The authors thank Drs. Honda and Yanai for providing viruses and Drs. Nakae and Flavell for providing mice. We also thank the members of the Atopy Research Center and the Department of Immunology for fruitful discussions and technical assistance, and Ms. Michiyo Matsumoto for secretarial assistance.

\section{References}

1 Galli SJ, Tsai M: Mast cells: versatile regulators of inflammation, tissue remodeling, host defense and homeostasis. J Dermatol Sci 2008;49:7-19.

2 Marshall JS, King CA, McCurdy JD: Mast cell cytokine and chemokine responses to bacterial and viral infection. Curr Pharm Des 2003;9:11-24.

-3 Sugiyama K: Histamine release from rat mast cells induced by Sendai virus. Nature 1977;270:614-615.

4 Patella V, Florio G, Petraroli A, Marone G: HIV-1 gp120 induces IL-4 and IL-13 release from human $\mathrm{Fc}$ epsilon $\mathrm{RI}^{+}$cells through interaction with the $\mathrm{VH} 3$ region of IgE. J Immunol 2000;164:589-595.
5 King CA, Anderson R, Marshall JS: Dengue virus selectively induces human mast cell chemokine production. J Virol 2002;76: 8408-8419.

6 Kulka M, Alexopoulou L, Flavell RA, Metcalfe DD: Activation of mast cells by doublestranded RNA: evidence for activation through Toll-like receptor 3. J Allergy Clin Immunol 2004;114:174-182.

7 Dietrich N, Rohde M, Geffers R, Kroger A, Hauser H, Weiss S, Gekara NO: Mast cells elicit proinflammatory but not type I interferon responses upon activation of TLRs by bacteria. Proc Natl Acad Sci USA 2010;107: 8748-8753.

-8 Orinska Z, Bulanova E, Budagian V, Metz M, Maurer M, Bulfone-Paus S: TLR3-induced activation of mast cells modulates $\mathrm{CD}^{+} \mathrm{T}$ cell recruitment. Blood 2005;106:978-987.
9 Stelekati E, Bahri R, D’Orlando O, Orinska Z, Mittrucker HW, Langenhaun R, Glatzel $\mathrm{M}$, Bollinger A, Paus R, Bulfone-Paus S: Mast cell-mediated antigen presentation regulates $\mathrm{CD}^{+} \mathrm{T}$ cell effector functions. Immunity 2009;31:665-676.

10 Yoneyama M, Fujita T: Recognition of viral nucleic acids in innate immunity. Rev Med Virol 2010;20:4-22.

11 Marshall JS: Mast-cell responses to pathogens. Nat Rev Immunol 2004;4:787-799.

-12 Supajatura V, Ushio H, Nakao A, Akira S, Okumura K, Ra C, Ogawa H: Differential responses of mast cell Toll-like receptors 2 and 4 in allergy and innate immunity. J Clin Invest 2002;109:1351-1359. 
13 Matsushima H, Yamada N, Matsue H, Shimada S: TLR3-, TLR 7-, and TLR 9-mediated production of proinflammatory cytokines and chemokines from murine connective tissue type skin-derived mast cells but not from bone marrow-derived mast cells. J Immunol 2004;173:531-541.

14 Alexopoulou L, Holt AC, Medzhitov R, Flavell RA: Recognition of double-stranded RNA and activation of NF-kappaB by Tolllike receptor 3. Nature 2001;413:732-738.

15 Kato H, Takeuchi O, Mikamo-Satoh E, Hirai R, Kawai T, Matsushita K, Hiiragi A, Dermody TS, Fujita T, Akira S: Length-dependent recognition of double-stranded ribonucleic acids by retinoic acid-inducible gene-I and melanoma differentiation-associated gene 5. J Exp Med 2008;205:1601-1610.

-16 Abraham SN, St John AL: Mast cell-orchestrated immunity to pathogens. Nat Rev Immunol 2010;10:440-452.

-17 St John AL, Rathore AP, Yap H, Ng ML, Metcalfe DD, Vasudevan SG, Abraham SN: Immune surveillance by mast cells during dengue infection promotes natural killer (NK) and NKT-cell recruitment and viral clearance. Proc Natl Acad Sci USA 2011;108:9190-9195.

-18 Edelmann KH, Richardson-Burns S, Alexopoulou L, Tyler KL, Flavell RA, Oldstone MB: Does Toll-like receptor 3 play a biological role in virus infections? Virology 2004; 322:231-238.

-19 Schulz O, Diebold SS, Chen M, Naslund TI, Nolte MA, Alexopoulou L, Azuma YT, Flavell RA, Liljestrom P, Reis e Sousa C: Toll-like receptor 3 promotes cross-priming to virusinfected cells. Nature 2005;433:887-892.
20 Brown MG, McAlpine SM, Huang YY, Haidl ID, Al-Afif A, Marshall JS, Anderson R: RNA sensors enable human mast cell antiviral chemokine production and IFN-mediated protection in response to antibody-enhanced dengue virus infection. PLoS One 2012;7:e34055.

21 Sumpter R Jr, Loo YM, Foy E, Li K, Yoneyama M, Fujita T, Lemon SM, Gale M Jr: Regulating intracellular antiviral defense and permissiveness to hepatitis C virus RNA rep lication through a cellular RNA helicase, RIG-I. J Virol 2005;79:2689-2699.

22 Yoneyama M, Kikuchi M, Natsukawa T, Shinobu N, Imaizumi T, Miyagishi M, Taira K, Akira S, Fujita T: The RNA helicase RIG-I has an essential function in double-stranded RNA-induced innate antiviral responses. Nat Immunol 2004;5:730-737.

23 Kawai T, Akira S: Innate immune recognition of viral infection. Nat Immunol 2006;7: 131-137.

24 Georgel P, Jiang Z, Kunz S, Janssen E, Mols J, Hoebe K, Bahram S, Oldstone MB, Beutler $B$ : Vesicular stomatitis virus glycoprotein $G$ activates a specific antiviral Toll-like receptor 4-dependent pathway. Virology 2007; 362:304-313.

25 Yoneyama M, Kikuchi M, Matsumoto K, Imaizumi T, Miyagishi M, Taira K, Foy E, Loo YM, Gale M Jr, Akira S, Yonehara S, Kato A, Fujita T: Shared and unique functions of the DExd/H-box helicases RIG-I, MDA5, and LGP2 in antiviral innate immunity. J Immunol 2005;175:2851-2858.
6 Kato H, Sato S, Yoneyama M, Yamamoto M, Uematsu S, Matsui K, Tsujimura T, Takeda K, Fujita T, Takeuchi O, Akira S: Cell typespecific involvement of RIG-I in antiviral response. Immunity 2005;23:19-28.

27 Lund JM, Alexopoulou L, Sato A, Karow M, Adams NC, Gale NW, Iwasaki A, Flavell RA: Recognition of single-stranded RNA viruses by Toll-like receptor 7. Proc Natl Acad Sci USA 2004;101:5598-5603.

28 Pichlmair A, Schulz O, Tan CP, Naslund TI, Liljestrom P, Weber F, Reis e Sousa C: RIGI-mediated antiviral responses to singlestranded RNA bearing 5'-phosphates. Science 2006;314:997-1001.

29 Kato H, Takeuchi O, Sato S, Yoneyama M, Yamamoto M, Matsui K, Uematsu S, Jung A, Kawai T, Ishii KJ, Yamaguchi O, Otsu K, Tsujimura T, Koh CS, Reis e Sousa C, Matsuura Y, Fujita T, Akira S: Differential roles of MDA5 and RIG-I helicases in the recognition of RNA viruses. Nature 2006;441:101105.

30 Hornung V, Ellegast J, Kim S, Brzozka K, Jung A, Kato H, Poeck H, Akira S, Conzelmann KK, Schlee M, Endres S, Hartmann G: 5'-Triphosphate RNA is the ligand for RIG-I. Science 2006;314:994-997.

31 Pichlmair A, Schulz O, Tan CP, Rehwinkel J, Kato H, Takeuchi O, Akira S, Way M, Schiavo G, Reis e Sousa C: Activation of MDA5 requires higher-order RNA structures generated during virus infection. J Virol 2009; 83:10761-10769.

32 Saito T, Gale M Jr: Principles of intracellular viral recognition. Curr Opin Immunol 2007; 19:17-23. 